\title{
EFFICIENT LARGE SCALE ANTENNA SELECTION BY PARTIAL SWITCHING CONNECTIVITY
}

\author{
Adrian Garcia-Rodriguez ${ }^{\dagger}$, Christos Masouros ${ }^{\star}$ and Pawel Rulikowski ${ }^{\dagger}$ \\ ${ }^{\dagger}$ Bell Laboratories, Nokia, Dublin, Ireland \\ * University College London (UCL), London, UK
}

\begin{abstract}
In this work we analyze the benefits of low-complexity radio frequency (RF) switching matrices (SMs) for antenna selection (AS) in large scale antenna systems (LSAS). The reduced RF complexity and insertion losses (ILs) are attained by limiting the number of internal connections in the SM, at the expense of a limited flexibility in the AS. The results presented in this paper demonstrate that partially-connected (PC) SMs outperform conventional fully-flexible (FF) alternatives due to their reduced ILs, which are characterized in this work.

Index Terms - Antenna selection, massive MIMO, RF switching matrices, insertion losses.
\end{abstract}

\section{INTRODUCTION}

Massive multiple-input multiple-output (MIMO) systems are one of the leading candidates for satisfying the spectral and energy efficiency objectives promulgated in 5G [1-3]. Specifically, LSAS propose to deploy base stations (BSs) comprised of tens to hundreds of antennas for providing high and stable data rates to a multiplicity of mobile terminals (MTs) [1-3]. However, the favourable propagation conditions attained by massive MIMO come at the expense of an increase in both the hardware and computational complexities of existing cellular systems, due to their excessive number of RF chains [2].

The fundamental idea behind AS implementations is to reduce the number of RF chains by judiciously activating a subset of antennas that maximizes performance $[4,5]$. In this line, numerous related works have proposed AS schemes with low computational complexity [4-7], characterized their energy efficiency gains [8,9], and described hardware-related issues that arise in their practical implementation $[10,11]$. The application of AS to massive MIMO systems has also constituted the focus of intense research efforts [9, 12-15]. Indeed, the results from the measurement campaigns carried out in $[12,15]$ motivate the deployment of AS in practical LSAS, since they demonstrate that the channels of separate antennas have distinct statistical characteristics.

In spite of the above research efforts, the implementation of SMs has attracted considerably less attention, although they constitute a critical component of AS systems $[4,10]$.

This work was supported by the Royal Academy of Engineering, UK and the EPSRC under grant EP/M014150/1.

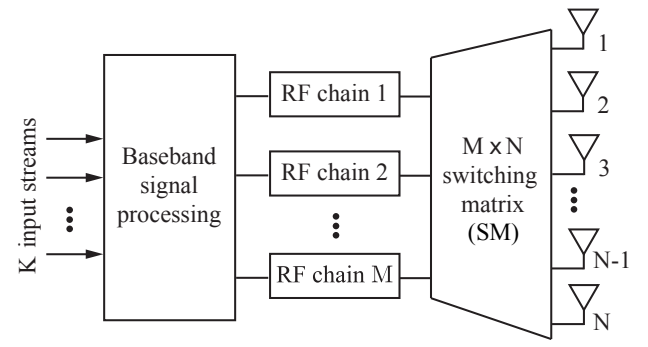

Fig. 1: Block diagram of the considered AS system.

Specifically, architectures for reducing the substantial hardware complexity and insertion losses (ILs) of SMs in massive MIMO have been recently promulgated both in [16], where independent groups of multiple antennas are connected to a single RF chain, and in [15], where each RF chain connects to a pair of antennas via binary RF switches. While these solutions are capable of reducing the power losses introduced by the RF switches, their actual performance gains have not been characterized because the internal architecture of SMs has not been comprehensively studied. For this reason, this paper concentrates on characterizing the impact of employing different SM hardware solutions on the performance of massive MIMO systems with AS, where the efficient design of RF switching components is crucial due to their considerable dimensions. Specifically, we analytically characterize the performance of power-based (PB) AS with PC SMs. Moreover, in contrast with prior literature, we define generalized optimization problems for AS with PC SMs in order to study the trade-off that arises in these systems: while their attainable performance is potentially reduced due to a limited switching flexibility, their reduced ILs, which are characterized in this work, provide simultaneous power gains for transmission.

\section{ANTENNA SELECTION AND RF SWITCHING MATRIX IMPLEMENTATIONS}

The model considered in the following is comprised of a BS with $N$ antennas and $K$ single-antenna MTs. The block diagram of the BS is illustrated in Fig. 1, where it can be observed that $M<N$ RF chains are implemented and, subsequently, only $M$ antennas are simultaneously active. The signal received at the MTs, $\mathbf{y} \in \mathbb{C}^{K \times 1}$, is given by

$$
\mathbf{y}=\sqrt{\rho K} \mathbf{H}_{[\mathcal{N}]} \mathbf{x}+\mathbf{n}=\sqrt{\bar{\rho} K / \delta} \mathbf{H}_{[\mathcal{N}]} \mathbf{x}+\mathbf{n},
$$



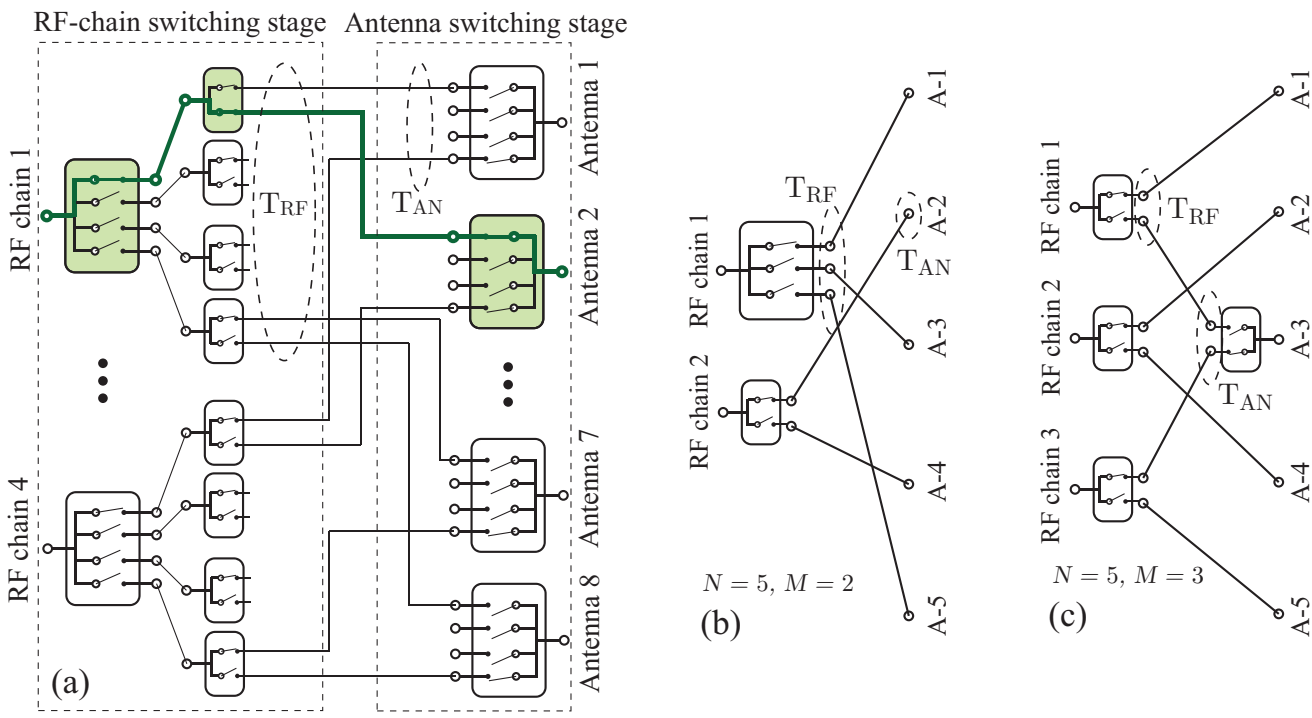

Fig. 2: Illustrative block diagrams for the RF switching matrices (SM) of a (a) FF $4 \times 8$ SM, (b) PC $2 \times 5$ SM and (c) PC $3 \times 5$ SM.

where $\mathbf{x} \in \mathbb{C}^{M \times 1}$ denotes the transmitted signal satisfying $\mathbb{E}\left[\mathbf{x}^{\mathrm{H}} \mathbf{x}\right]=1, \mathbf{n} \in \mathbb{C}^{K \times 1}$ is the circularly symmetric additive white-Gaussian noise vector with its $k$-th entry following $n_{k} \sim \mathcal{C N}(0,1)$, and $\mathbf{H} \in \mathbb{C}^{K \times N}$ collects the coefficients characterizing the frequency channel response between the BS and the MTs. Here, $\mathbf{H}_{[\mathcal{M}]} \in \mathbb{C}^{K \times M}$ is a submatrix of $\mathbf{H}$ constructed by choosing the columns specified by the set $\mathcal{M}$ of cardinality $|\mathcal{M}|=M$, which represents the selected antennas. Moreover, $\bar{\rho}$ represents the average transmission power per MT without accounting for the ILs introduced by the SM, $\delta$, which are characterized in the following. Note that (1) considers that the RF SM is placed after the power amplifiers (PAs) in the transmission chain, which corresponds to the hardware solution that minimizes the number of analog hardware components [4].

\subsection{Fully-Flexible (FF) RF Switching Matrices}

In this work we concentrate on the AS with full switching flexibility that maximizes capacity by solving $[12,17]$

$$
\begin{aligned}
\mathcal{P}_{1}: \underset{\mathbf{S}, \mathbf{P}}{\operatorname{maximize}} & \log _{2} \operatorname{det}\left(\mathbf{I}_{K}+\rho K \mathbf{P} \mathbf{H S H} \mathbf{H}^{\mathrm{H}}\right) \\
\text { subject to } & \sum_{i=1}^{N} S_{i, i}=M, \\
& 0 \leq S_{n, n} \leq 1, \forall n \in\{1, \ldots, N\},
\end{aligned}
$$

where $\mathbf{P} \in \mathbb{R}^{K \times K}$ is a diagonal matrix characterizing the power allocation over the different streams $\left(\sum_{i=1}^{K} P_{i, i}=1\right)$ and $\mathbf{S} \in \mathbb{B}^{N \times N}$ is a diagonal matrix with $S_{n, n}=1, n \in$ $\{1, \ldots, N\}$ if the $n$-th antenna is selected and $S_{n, n}=0, n \in$ $\{1, \ldots, N\}$, otherwise. Due to the difficulty of jointly optimizing over both $\mathbf{S}$ and $\mathbf{P}$ in $\mathcal{P}_{1}$ and similarly to $[12,15], \mathbf{S}$ is firstly obtained assuming $\mathbf{P}=\mathbf{I}_{K}$ and, subsequently, $\mathbf{P}$ is computed by solving $\mathcal{P}_{1}$ with $\mathbf{S}$ as a given matrix.

The SMs that implement the AS in $\mathcal{P}_{1}$ are comprised of primary switches with a smaller number of input and output
Table 1: Primary switches

\begin{tabular}{|l|l|l|}
\hline Switch type & Model $[18]$ & Insertion loss \\
\hline \hline $1 \times 2$ ( 2 output ports) & PE42422 & $L_{2}=0.25 \mathrm{~dB}$ \\
\hline $1 \times 3$ ( 3 output ports) & PE42430 & $L_{3}=0.45 \mathrm{~dB}$ \\
\hline $1 \times 4$ ( 4 output ports) & PE42440 & $L_{4}=0.45 \mathrm{~dB}$ \\
\hline
\end{tabular}

ports, such as those detailed in Table $1[19,20]$. Fig. 2(a) represents the architecture of an illustrative FF SM, which is comprised of two switching sub-stages represented by dashed boxes: the RF-chain and the antenna switching stages $[19,20]$. These switching stages are constructed by concatenating primary switches of smaller size such as those described in Table 1 . Therefore, determining the number of primary switches implemented is required for characterizing the total ILs ( $\delta$ in (1)) introduced in the switching procedure. With this purpose, let us denote $T_{\mathrm{AN}}$ and $T_{\mathrm{RF}}$, which are represented in Fig. 2, as the maximum number of ports per antenna in the antenna in the antenna switching stage or per RF chain in the RF-chain switching stage, respectively. For FF architectures, it can be shown that these are given by

$$
T_{\mathrm{RF}}=N \text {, and } T_{\mathrm{AN}}=M .
$$

Let us also define $\mathcal{T}$ as a (decreasingly) ordered set with cardinality $|\mathcal{T}| \triangleq N_{\mathrm{s}}$ containing the number of output ports in the primary switches, and $\delta_{\mathcal{T}_{j}}$ as the ILs in $\mathrm{dB}$ of a primary switch with $\mathcal{T}_{j}$ output ports. For instance, $\mathcal{T}=\{4,3,2\}, N_{\mathrm{s}}=3$ and $S_{\{2,3,4\}}=\{0.25,0.45,0.45\} \mathrm{dB}$ for the primary switches in Table 1. Therefore, the ILs introduced by an arbitrary SM architecture can be expressed as

$$
\delta_{\mathrm{dB}}=\sum_{j=1}^{N_{\mathrm{s}}}\left(S_{\mathcal{T}_{j}}^{\mathrm{RF}}+S_{\mathcal{T}_{j}}^{\mathrm{AN}}\right) \times \delta_{\mathcal{T}_{j}} .
$$

Here, $S_{\left.\mathcal{T}_{j}, \mathrm{AN}\right\}}^{\{\mathrm{RF}}$ denote the number of consecutive primary switches with $\mathcal{T}_{j}$ output ports that the signals cross in the RF- 
chain (or antenna) switching stages, which can be iteratively computed as

$$
S_{\mathcal{T}_{j}}^{\{\mathrm{RF}, \mathrm{AN}\}}=\operatorname{fact}\left(Q_{\mathcal{T}_{j}}^{\{\mathrm{RF}, \mathrm{AN}\}}, \mathcal{T}_{j}\right), j \in\left\{1, \ldots, N_{\mathrm{s}}\right\},
$$

where fact $(a, b)$ denotes the number of times that $b$ arises in the integer factorization of $a$ and $Q_{\mathcal{T}_{j}}^{\{\mathrm{RF}, \mathrm{AN}\}}$ can be expressed as

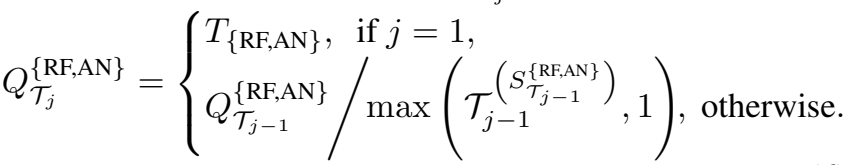

Note that $\mathcal{T}_{j}^{S \mathcal{T}_{j}}$ in (6) denotes the number of output ports that can be obtained by concatenating $S_{\mathcal{T}_{j}}$ switching stages comprised of primary switches with $\mathcal{T}_{j}$ output ports. For instance, it can be shown by application of the above expressions that for an RF switching stage with $T_{\mathrm{RF}}=Q_{4}^{\mathrm{RF}}=N=8$ such as that shown in Fig. 2(a), the transmit signal must cross $S_{4}^{\mathrm{RF}}=$ fact $(8,4)=$ fact $(4 \times 2,4)=1$ primary switch with $\mathcal{T}_{1}=4$ output ports and $S_{2}^{\mathrm{RF}}=1$ switch with $\mathcal{T}_{3}=2$ output ports, as coloured in Fig. 2(a). To reduce the number of internal switches and the total ILs, $\delta_{\mathrm{dB}}$, in the following we consider an RF switching architecture that achieves both objectives by limiting the input-output connectivity of the SM.

\subsection{Partially-Connected (PC) RF Switching Matrices}

The PC architecture is designed to minimize the number of primary switches while guaranteeing that each antenna is connected to at least one RF chain, i.e.

$$
T_{\mathrm{RF}}=\left\lceil\frac{N}{M}\right\rceil, \text { and } T_{\mathrm{AN}}= \begin{cases}1, & \text { if }\left\lfloor\frac{N}{M}\right\rfloor \geq 2, \\ 2, & \text { otherwise. }\end{cases}
$$

The reduced number of ports in the RF-chain and switching stages entails that PC switching architectures introduced reduced ILs when compared with their FF counterparts. Illustrative examples of these architectures are represented in Fig. 2(b) and (c) for the cases of $2 \times 5$ and $3 \times 5$ SMs, respectively.

Remark: It would seem intuitive that increasing the number of RF chains should enhance the performance of AS systems. However, it should be noted that this might come at the cost of incorporating additional primary switches to the SM, hence introducing additional ILs that might degrade the overall performance. For instance, when compared with the SM of Fig. 2(b) with $M=2$, the SM of Fig. 2(c) $(M=3)$ requires an additional primary switch at the antenna switching stage. The study of this trade-off constitutes the focus of Sec. 4.

Clearly, $\mathcal{P}_{1}$ should account for the restrictive switching connectivity of PC SMs. Motivated by [15] and in order to incorporate these additional constraints, in the following we consider that the overall antenna array is divided into subarrays. These subarrays are constructed by connecting each RF chain to antenna ports as physically separated as possible, with the purpose of enhancing spatial diversity. The distance between these antenna ports, $N_{\text {dist }}$, can be expressed as

$$
N_{\text {dist }}=\left\{\begin{array}{l}
M, \text { if }\lfloor N / M\rfloor \geq 2, \\
N-M, \text { otherwise. }
\end{array}\right.
$$

For example, Fig. 2(c) shows that each RF chain connects to alternative antenna ports, i.e. $N_{\text {dist }}=N-M=2$. Moreover, let $S_{\text {sub }}$ denote the number of independent antenna subarrays, which do not share any common RF chain. The antenna elements comprising the $i$-th independent subarray $\left(i \in\left\{1, \ldots, S_{\text {sub }}\right\}\right)$ are therefore given by

$\mathcal{N}^{i}=\left\{i, i+N_{\mathrm{dist}}, \ldots, i+\left(\left\lceil\frac{N-i+1}{N_{\mathrm{dist}}}\right\rceil-1\right) N_{\mathrm{dist}}\right\}$,

where each RF chain connects to antennas with indices separated by $N_{\text {dist }}$. Similarly, let $\left|\mathcal{M}^{i}\right|$ denote the number of antennas that can be simultaneously active in the $i$-th subarray, which corresponds to the number of RF chains connected to that subarray. By considering the above, the general convex optimization problem with partial connectivity constraints can be formulated as

$$
\begin{aligned}
& \mathcal{P}_{2}: \underset{\mathbf{S}, \mathbf{P}}{\operatorname{maximize}} \log _{2} \operatorname{det}\left(\mathbf{I}_{K}+\rho K \mathbf{P} \mathbf{H S H} \mathbf{H}^{\mathrm{H}}\right) \\
& \text { subject to } \sum_{i=1}^{N} S_{i, i}=M, \\
& 0 \leq S_{i, i} \leq 1, \forall i \in\{1, \ldots, N\}, \\
& \sum_{j \in \mathcal{N}^{i}} S_{j, j}=\left|\mathcal{M}^{i}\right|, \forall i \in\left\{1, \ldots, S_{\mathrm{sub}}\right\} .
\end{aligned}
$$

\section{PERFORMANCE ANALYSIS}

The expressions developed in this section leverage upon the ergodic capacity approximation derived in [21] for powerbased (PB)-AS systems ${ }^{1}$, which is given by

$$
C_{\mathrm{PS}-\mathrm{PC}} \approx \mathbb{E}_{\mathbf{G}}\left[\log _{2} \operatorname{det}\left(\mathbf{I}_{K}+\rho K \widetilde{P}_{\mathrm{PC}} \mathbf{G G}^{\mathrm{H}}\right)\right],
$$

where the entries of $\mathbf{G} \in \mathbb{C}^{K \times M}$ follow the same distribution of those from $\mathbf{H}$ and, similarly to [21, Eq. 9], we propose to express $\widetilde{P}_{\mathrm{PC}}$ for the case of PC switching architectures as

$$
\widetilde{P}_{\mathrm{PC}}=\frac{1}{K M} \sum_{j=1}^{\left(\begin{array}{l}
N \\
M
\end{array}\right)}\left(\sum_{i=1}^{M} \mathbb{E}_{\mathbf{H}}\left[B_{\mathcal{B}_{i}^{j}: N}\right]\right) \times P\left(T_{j}\right) .
$$

Here, $\mathcal{B}^{j}, j \in\left\{1, \ldots,\left(\begin{array}{l}N \\ M\end{array}\right)\right\}$ are sets with cardinality $\left|\mathcal{B}^{j}\right|=$ $M$ that comprise a given combination of ordered column norms of $\mathbf{H}$, whereas $P\left(T_{j}\right)$ denotes the probability of selecting the specific combination of columns of $\mathbf{H}$ determined by $\mathcal{B}^{j}$. Moreover, $\mathcal{B}_{i}^{j}$ denotes the $i$-th entry of $\mathcal{B}^{j}$, whereas $B_{i: N}$ represents the $i$-th smallest column norm $\left(\left\|\mathbf{h}_{c}\right\|^{2} \triangleq\right.$ $\left.\sum_{j}^{K}\left|h_{c, j}\right|^{2}\right)$ of $\mathbf{H}$, i.e. $\left\{B_{1: N}<B_{2: N}<\cdots<B_{N: N}\right\}$. At this point it should be noted that, when compared with [21, Eq. 9], the binary random variables $T_{j} \in\{0,1\}$, which

\footnotetext{
${ }^{1}$ This scheme selects the antennas with largest channel power and constitutes our focus because it approximates the performance of the schemes relying on instantaneous channel state information (CSI), which instead require additional time-frequency resources for accurate CSI acquisition [22].
} 
determine the probability of selecting or not the ordered combination $\mathcal{B}^{j}$, are introduced to account for the fact that the combination corresponding to the antennas corresponding with the largest columns norms might not be selected, due to the limited switching connectivity.

Overall, since the result of the expectation in (11) is already available for multiple correlated and uncorrelated communication channels [22], our focus is placed on both $\mathbb{E}_{\mathbf{H}}\left[B_{\mathcal{B}_{i}^{j}: N}\right]$ and $P\left(T_{j}\right)$ in (12), which have to be determined for computing the ergodic capacity. Specifically, since $B_{\mathcal{B}_{i}^{j}: N}$ represents the $\mathcal{B}_{i}^{j}$-th smallest column norm of $\mathbf{H}$, conventional ordered statistics can be employed for computing $\mathbb{E}_{\mathbf{H}}\left[B_{\mathcal{B}_{i}^{j}: N}\right]$ under different distributions such as those of Rayleigh flat-fading channels, i.e. [23]

$$
\begin{gathered}
\mathbb{E}_{\mathbf{H}}\left[B_{t: N}\right]=\frac{N !}{(M-1) !(t-1) !(N-t) !} \sum_{r=0}^{t-1}(-1)^{r}\left(\begin{array}{c}
t-1 \\
r
\end{array}\right) \\
\times \sum_{s=0}^{(K-1)(N-t+r)} a_{s}(N-t+r) \frac{(K+s) !}{(N-t+r+1)^{K+s+1}},
\end{gathered}
$$

where $a_{s}(N-t+r)$ denotes the polynomial coefficient of $x^{s}$ in $\left(\sum_{s}^{K-1} \frac{x^{s}}{s !}\right)^{N}$. Moreover, although not explicitly shown here for reasons of space, the computation of $P\left(T_{j}\right)$ is tractable for channels without correlation at the BS $[1,24]$. Intuitively, these probabilities can be derived by noting that the probability of finding the ordered statistic $B_{\mathcal{B}_{i}^{j}: N}$ at a given antenna port is identical for all antennas when the above assumption is adopted, as detailed in [24].

\section{SIMULATION RESULTS}

This section presents numerical results for characterizing the trade-off that arises when PC SMs are considered, i.e., while they perform a suboptimal AS, they might still provide overall performance gains due to their reduced ILs. For this, a frequency-flat Rayleigh fading channel is considered, i.e. $\mathbf{H} \sim \mathcal{C N}\left(\mathbf{0}, \mathbf{I}_{K} \otimes \mathbf{I}_{N}\right)$. We consider both AS relying on the instantaneous CS via $\mathcal{P}_{\{1,2\}}$, and on selecting the antennas according to their channel power (PB-AS) as per Sec. $3[4,21]$.

Fig. 3 illustrates both the simulated and theoretical spectral efficiencies (SEs) of a small scale AS system with $N=$ $10, K=2$ and $\bar{\rho}=10 \mathrm{~dB}$, while accounting for the ILs as per (4). The results of this figure demonstrate that implementing a larger number of RF chains is generally beneficial for enhancing the system's SE. Note that this occurs in spite of the increase of the switching losses with $M$. Fig. 3 also shows that PC-AS is able to outperform FF-AS, due to their smaller power losses. It can also be observed that the theoretical approach of Sec. 3 for PB-AS closely matches the simulation results, hence validating our analysis.

Fig. 4 concentrates on showing the evolution of the SE against $M$ in an LSAS with $N=64, K=8$ and $\bar{\rho}=10$

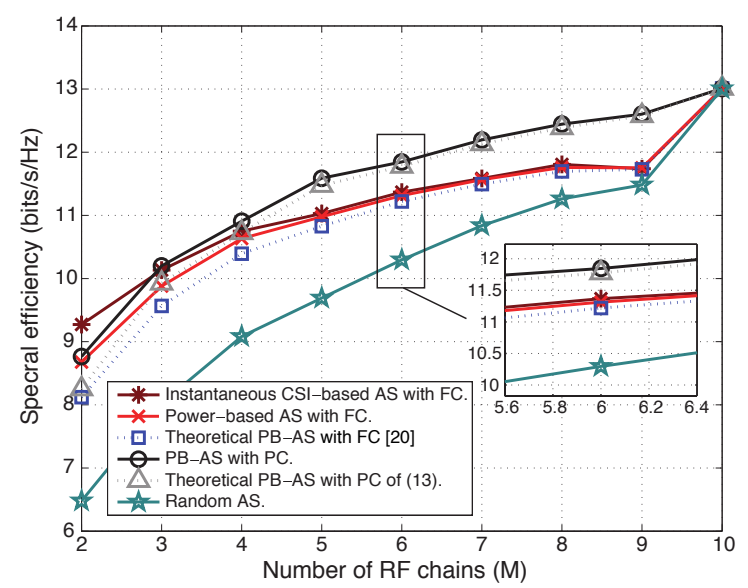

Fig. 3: Spectral efficiency (bits/s/Hz) vs. $M$ with FF and PC switching matrices. $N=10, K=2$ and $\bar{\rho}=10 \mathrm{~dB}$.

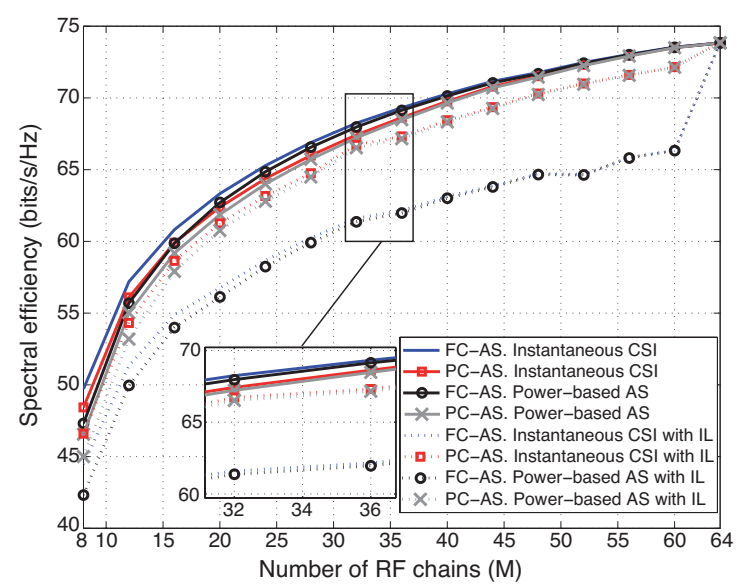

Fig. 4: Spectral efficiency (bits/s/Hz) vs. $M$ with FF and PC switching matrices. $N=64, K=8$ and $\bar{\rho}=10 \mathrm{~dB}$.

$\mathrm{dB}$. The performance both with ( $\delta$ in (1) defined as per (4)) and without $(\delta=1)$ accounting for the switching ILs are represented. Fig. 4 shows that there exist negligible performance differences between the strategies considered in this paper when the switching ILs are ignored. This is because, while not being able to select the optimal antenna combination, PC-AS is still capable of selecting other combination with a similar performance due to the large $N$. As shown in Fig. 4, this entails that PC-AS can provide significant performance benefits in the LSAS regime when compared with their FF counterparts when the switching losses are incorporated, due to their simplified switching architecture and reduced ILs.

\section{CONCLUSION}

In this paper, the impact of considering different hardware implementations for the RF SMs required AS systems has been studied. We have characterized the ILs introduced by different SMs as well as the performance loss they introduce in practical AS systems. Altogether, the analytical and simulation results obtained in this work prompt the implementation of PC switching matrices in realistic AS implementations. 


\section{REFERENCES}

[1] F. Rusek et al., "Scaling up MIMO: Opportunities and challenges with very large arrays," IEEE Signal Process. Mag., vol. 30, no. 1, pp. 40-60, Jan. 2013.

[2] E. Björnson, E. G. Larsson, and T. L. Marzetta, "Massive MIMO: Ten myths and one critical question," IEEE Commun. Mag., vol. 54, no. 2, pp. 114-123, Feb. 2016.

[3] Lu Lu et al., "An overview of massive MIMO: Benefits and challenges," IEEE Journal of Sel. Topics in Signal Process., vol. 8, no. 5, pp. 742-758, Oct. 2014.

[4] A. F. Molisch and M. Z. Win, "MIMO systems with antenna selection," IEEE Microwave Mag., vol. 5, no. 1, pp. 46-56, March 2004.

[5] S. Sanayei and A. Nosratinia, "Antenna selection in MIMO systems," IEEE Commun. Mag., vol. 42, no. 10, pp. 68-73, Oct. 2004.

[6] A. Gorokhov, D. A. Gore, and A. J. Paulraj, "Receive antenna selection for MIMO spatial multiplexing: Theory and algorithms," IEEE Trans. on Signal Process., vol. 51, no. 11, pp. 2796-2807, Nov. 2003.

[7] A. Dua, K. Medepalli, and A. J. Paulraj, "Receive antenna selection in MIMO systems using convex optimization," IEEE Trans. on Wireless Commun., vol. 5, no. 9, pp. 2353-2357, Sept. 2006.

[8] N. P. Le, F. Safaei, and L. C. Tran, "Antenna selection strategies for MIMO-OFDM wireless systems: An energy efficiency perspective," IEEE Trans. on Veh. Tech., vol. 65, no. 4, pp. 2048-2062, April 2016.

[9] Hui Li, Lingyang Song, and M. Debbah, "Energy efficiency of large-scale multiple antenna systems with transmit antenna selection," IEEE Trans. on Commun., vol. 62, no. 2, pp. 638-647, Feb. 2014.

[10] A. F. Molisch et al., "Implementation aspects of antenna selection for MIMO systems," in Int. Conf. on Commun. and Networking in China, Oct. 2006, pp. 1-7.

[11] J. Ahmadi-Shokouh, S. H. Jamali, S. Safavi-Naeini, and G. Z. Rafi, "Switch loss and antenna directivity effects on MIMO antenna selection," in Canadian Conf. on Electrical and Computer Engineering (CCECE), May 2008, pp. 641-646.

[12] Xiang Gao, O. Edfors, F. Tufvesson, and E. G. Larsson, "Massive MIMO in real propagation environments: Do all antennas contribute equally?," IEEE Trans. on Commun., vol. 63, no. 11, pp. 3917-3928, Nov. 2015.
[13] M. Gkizeli and G. N. Karystinos, "Maximum-SNR antenna selection among a large number of transmit antennas," IEEE Journal of Sel. Topics in Signal Process., vol. 8, no. 5, pp. 891-901, Oct. 2014.

[14] P. V. Amadori and C. Masouros, "Interference driven antenna selection for massive multi-user MIMO," IEEE Trans. on Vehicular Technology, vol. PP, 2015.

[15] X. Gao, O. Edfors, F. Tufvesson, and E. G. Larsson, "Multi-switch for antenna selection in massive MIMO," in IEEE Global Commun. Conf. (GLOBECOM), Dec. 2015, pp. 1-6.

[16] R. Méndez-Rial et al., "Hybrid MIMO architectures for millimeter wave communications: Phase shifters or switches?," IEEE Access, vol. 4, pp. 247-267, 2016.

[17] A. F. Molisch, M. Z. Win, Yang-Seok Choi, and J. H. Winters, "Capacity of MIMO systems with antenna selection," IEEE Trans. on Wireless Commun., vol. 4, no. 4, pp. 1759-1772, July 2005.

[18] Peregrine semiconductor, "Portfolio of RF switches," [Online]. Available: http://www.psemi.com/products/rfswitches.

[19] Teledyne Coax Switches, "Coax switch matrix selection guide," 2014, [Online]. Available: http://www.teledynecoax.com/pdf/csm_selection _guide.pdf.

[20] Sara Nazemzadeh, "A guide to RF switching systems," The RF \& Microwave Solutions Update, pp. 34-44, June 2008.

[21] P. J. Smith, T. W. King, L. M. Garth, and M. Dohler, "A power scaling analysis of norm-based antenna selection techniques," IEEE Trans. on Wireless Commun., vol. 7, no. 8, pp. 3140-3149, Aug. 2008.

[22] A. B. Narasimhamurthy and C. Tepedelenlioglu, "Antenna selection for MIMO-OFDM systems with channel estimation error," IEEE Trans. on Vehicular Technology, vol. 58, no. 5, pp. 2269-2278, June 2009.

[23] Shanti S Gupta, "Order statistics from the gamma distribution," Technometrics, vol. 2, no. 2, pp. 243-262, 1960 .

[24] A. Garcia-Rodriguez, C. Masouros, and P. Rulikowski, "Reduced switching connectivity for powerefficient large scale antenna selection," submitted to IEEE Trans. on Commun.. [Online]. Available: http://arxiv.org/abs/1605.01549. 